\title{
On TRACK: Primary Care Opportunities for Filling Unmet Need
}

\author{
Kurt C. Stange, $M D, P b D$, Editor
}

Ann Fam Med 2007;5:270-272. DOI: 10.1370/afm.712.

\section{EXPANDED PRIMARY CARE CAN FILL CRITICAL HOLES IN HEALTH CARE}

The study of outpatient treatment of opioid addiction in the last issue of Annals ${ }^{1}$ stimulated a dialogue between the lead author ${ }^{2-4}$ and an international group of clinicians sharing their experience with buprenorphine-naloxone and relevant literature, pharmacology, and politics. ${ }^{5-8}$ Together, these comments point the way toward expanded use, training, and research on this important intervention in primary care.

The study of a primary care response to Hurricane Katrina $^{9}$ kindled sharing of a parallel response from those caring for evacuees in the Houston Astrodome. ${ }^{10}$ Commentary from public health and emergency care experts $^{11-13}$ draws the larger lessons about the holes in the emergency response system and the vital role of primary care in meeting emergency needs. "This pragmatic study needs to be read by every communitybased department of health and disaster planner." ${ }^{\prime \prime 3}$

Coyne $^{14}$ challenges an inference by Gaynes et al that depressed patients should be treated as aggressively in primary care as in psychiatric care. ${ }^{15}$ He calls for reinterpretation of "potentially misleading implications," as well as further research on representative patient samples using semistructured interviews for diagnosis of depression.

Keller's discussion ${ }^{16}$ of the Wadland study ${ }^{17}$ linking primary care practice with quit lines provides evidence and expert interpretation of this promising and challenging strategy for expanding tobacco control.

The risk adjustment models used by James and colleagues $^{18}$ generated gratitude for their utility in preventing those providing care by rural hospitals from being unjustifiably maligned ${ }^{19,20}$ and detailed discussion of the challenges of adjusting for confounding. ${ }^{21,22}$

\section{DTC PHARMACEUTICAL MARKETING}

The discussion of direct to consumer (DTC) marketing started by the study by Frosch and colleagues ${ }^{23}$ con- tinued to percolate, further stimulated by an editorial ${ }^{24}$ and a news item from one of the Annals' sponsoring organizations in the last issue. ${ }^{25}$ Among several discussants, ${ }^{26,27}$ Krueger notes the conflict of interest of a wide variety of media outlets that have become dependent on DTC pharmaceutical ads, yet are responsible for reporting adverse effects of drugs as part of their news reporting. Frey suggests that DTC advertising bans could be a potent portion of presidential candidates' health care plans. ${ }^{28}$ Shropshire calls for redirecting American Academy of Family Physicians (AAFP) leadership away from support for the pharmaceutical industry toward "our patients' ultimate well-being...."29 Rather than banning DTC ads, ${ }^{30}$ Hallgren suggests that "our lobbying bodies (AAFP, American Medical Association) should be campaigning against it and providing the outlets of unbiased information."

\section{OTHER THREADS OF DISCUSSION}

Diverse discussants of research capacity building ${ }^{32-35}$ bring out multiple opportunities for stimulating primary care research, while challenging us to examine the impact of that research. ${ }^{36}$

The discussion of the study by Krist et $\mathrm{al}^{37}$ advances our understanding of the appropriate outcomes for use of patient decision aids. ${ }^{38,39}$

The study of standardized patients ${ }^{40}$ raises important questions ${ }^{41}$ and experiential validation and interpretation of this method ${ }^{42-44}$ of growing importance for health care research.

The challenge and need for expanding clinical performance measurement ${ }^{45}$ is well articulated in discussion by Milstein, ${ }^{46}$ de Brantes ${ }^{47}$ and Bennett. ${ }^{48}$ These calls for increased rigor in performance assessment are echoed somewhat differently in reaction to the essay on jazz as a metaphor for the art of improvisation in the medical encounter. ${ }^{49}$ This essay stimulated analysis and experience-based reflection, ${ }^{50,51}$ and a call for increased training in the "seventh competency" of reflective practice. ${ }^{52}$ 
Please join the exchange of ideas at http://www. AnnFamMed.org.

\section{References}

1. Mintzer IL, Eisenberg M, Terra M, MacVane C, Himmelstein D, Woolhandler S. Treating opioid addiction with buprenorphine-naloxone in community-based primary care settings. Ann Fam Med. 2007;5(2):146-150

2. Mintzer IL. Relapse and drug diversion [eletter]. http://www. annfammed.org/cgi/eletters/5/2/146\#5515, 28 March 2007.

3. Mintzer IL. Antagonist/antagonist roles [eletter]. http://www. annfammed.org/cgi/eletters/5/2/146\#55732 April 2007.

4. Mintzer IL. Dispensing buprenorphine/naloxone [eletter]. http:// www.annfammed.org/cgi/eletters/5/2/146\#5668, 30 April 2007.

5. Blondell RD. Data for evidence-based practice guidelines are needed [eletter]. http://www.annfammed.org/cgi/eletters/5/2/146\#5489 27 March 2007.

6. Albanese MJ. Opioid addiction treatment in primary care practice [eletter]. http://www.annfammed.org/cgi/eletters/5/2/146\#5512, 28 March 2007.

7. Fiscella K. Buprenorphine is underused by primary care physicians [eletter]. http://www.annfammed.org/cgi/eletters/5/2/146\#5520, 28 March 2007.

8. Hall RH. Routine family practice has treated fifty patients with suboxone [eletter]. http://www.annfammed.org/cgi/eletters/5/2/146\#5653, 26 April 2007.

9. Edwards TD, Young R, Lowe AF. Caring for a surge of hurricane Katrina evacuees in primary care clinics. Ann Fam Med. 2007;5(2):170-174

10. Gavagan TF. Ongoing disaster response preparedness concerns post-Katrina [eletter]. http://www.annfammed.org/cgi/eletters/5/2/170\#5585, 4 April 2007

11. Higgins W. Lessons from the JPS model [eletter]. http://www. annfammed.org/cgi/eletters/5/2/170\#5568, 2 April 2007.

12. Hick JL. The power of primary care [eletter]. http://www. annfammed.org/cgi/eletters/5/2/170\#5507, 28 March 2007.

13. Burkle FM. Exposing vulnerabilities: primary health care and surge capacity [eletter]. http://www.annfammed.org/cgi/eletters/5/2/170\#5492, 27 March 2007.

14. Coyne JC. Disseminating misleading information about major depression in primary care [eletter]. http://www.annfammed.org/ cgi/eletters/5/2/126\#5576, 4 April 2007.

15. Gaynes BN, Rush A, Trivedi M, et al. Major depression symptoms in primary care and psychiatric care settings: a cross-sectional analysis. Ann Fam Med. 2007;5(2):126-134.

16. Keller PA. Performance feedback holds promise for integrating evidence-based tobacco treatment into family practice [eletter]. http:// www.annfammed.org/cgi/eletters/5/2/135\#5636, 23 April 2007.

17. Wadland WC, Holtrop J, Weismantel D, Pathak P, Fadel H, Powell J. Practice-based referral rates to a tobacco cessation quit line: assess ing the impact of comparative feedback vs general reminders. Ann Fam Med. 2007;5(2):135-142.

18. James PA, Li P, Ward M. Myocardial infarction mortality in rural and urban hospitals: rethinking measures of quality of care. Ann Fam Med. 2007;5(2):105-111.

19. Longenecker R. Rural contributes to the quality discussion [eletter]. http://www.annfammed.org/cgi/eletters/5/2/105\#5527, 28 March 2007.

20. Vinson DC. Thanks for a careful analysis of a challenging topic [eletter]. http://www.annfammed.org/cgi/eletters/5/2/105\#5624, 14 April 2007.
21. Oliver MN. Risk adjustment: old methods must change [eletter]. http://www.annfammed.org/cgi/eletters/5/2/105\#5583, 4 April 2007

22. James PA. Author's response [eletter]. http://www.annfammed.org/ cgi/eletters/5/2/105\#5587, 6 April 2007.

23. Frosch DL, Krueger P, Hornik R, Barg F, Cronholm P. Creating demand for prescription drugs: a content analysis of television direct-to-consumer-advertising. Ann Fam Med. 2007;5(1):6-7.

24. Stange KC. Time to ban direct-to-consumer prescription drug marketing. Ann Fam Med. 2007;5(2):101-104

25. Kellerman R. AAFP supports improvement, not ban, on direct-toconsumer prescription drug ads. Ann Fam Med. 2007;5(2):180-181.

26. Alevizos AG. Effects of DTC advertising [eletter]. http://www. annfammed.org/cgi/eletters/5/1/6\#5630, 17 April 2007.

27. Noon S. Creating new patient population [eletter]?? http://www. annfammed.org/cgi/eletters/5/1/6\#5477, 21 March 2007.

28. Frey JJ. Selling drugs [eletter]. http://www.annfammed.org/cgi/eletters/5/1/6\#5245, 11 February 2007

29. Shropshire JH. Disappointment with AAFP leadership on direct to consumer ads [eletter]. http://www.annfammed.org/cgi/eletters/5/2/180\#5503, 27 March 2007.

30. Rafool G. Direct to consumer ads are a disgrace to medicine [eletter]. http://www.annfammed.org/cgi/eletters/5/2/101\#5509, 28 March 2007.

31. Hallgren JD. Dissenting opinion [eletter]. http://www.annfammed org/cgi/eletters/5/2/101\#5646, 23 April 2007.

32. Mahoney MC. Mahoney MC, Verma P, Morantz S. Research produc tivity among recipients of AAFP foundation grants. Ann Fam Med. 2007;5(2):143-145

33. Longo DR. Building research capacity in family medicine: various strategies are required [eletter]. http://www.annfammed.org/cgi/ eletters/5/2/143\#5658, 30 April 2007.

34. Diamond J]. An enviable record [eletter]. http://www.annfammed org/cgi/eletters/5/2/143\#5655, 28 April 2007.

35. Rosemann T. Grant programs for general practice in Germany [eletter]. http://www.annfammed.org/cgi/eletters/5/2/143\#5649, 24 April 2007

36. Askew D. What counts when you measure research productivity? [eletter]. http://www.annfammed.org/cgi/eletters/5/2/143\#5633, 17 April 2007.

37. Krist AH, Woolf $\mathrm{SH}$, Johnson R, Kerns J. Patient education on prostate cancer screening and involvement in decision making. Ann Fam Med. 2007;5(2):112-119.

38. Pignone MP. The effect of a decision aid on patient participation in decision making [eletter]. http://www.annfammed.org/cgi/eletters/5/2/112\#5571, 2 April 2007

39. McNutt R. Shared decision making [eletter]. http://www. annfammed.org/cgi/eletters/5/2/112\#5581, 4 April 2007.

40. Fiscella K, Franks P, Srinivasan M, Kravitz R, Epstein RM. Ratings of physician communication by real and standardized patients. Ann Fam Med. 2007:5(2):151-158.

41. Mauksch LB. More questions than answers-evidence of study value [eletter]. http://www.annfammed.org/cgi/eletters/5/2/151\#5617, 14 April 2007.

42. Carney PA. Important standardized patient research [eletter]. http:// www.annfammed.org/cgi/eletters/5/2/151\#5494, 27 March 2007.

43. Fiscella K. Unique perspective of standardized patients [eletter]. http://www.annfammed.org/cgi/eletters/5/2/151\#5517, 28 March 2007.

44. Howley LD. Trained evaluators increase validity of outcomes [eletter]. http://www.annfammed.org/cgi/eletters/5/2/151\#5535, 29 March 2007. 
45. Werner RM, Asch D. Clinical concerns about clinical performance measurement. Ann Fam Med. 2007;5(2):159-163.

46. Milstein AS. Rather than deemphasize measures, measure what's important [eletter]. http://www.annfammed.org/cgi/eletters/5/2/159\#5542, 29 March 2007.

47. de Brantes FS. Measuring the hard way [eletter]. http://www. annfammed.org/cgi/eletters/5/2/159\#5504, 27 March 2007.

48. Bennett IM. Quality measures may require more time [eletter]. http://www.annfammed.org/cgi/eletters/5/2/159\#5498, 27 March 2007.
49. Haidet P. Jazz and the 'art' of medicine: improvisation in the medical encounter. Ann Fam Med. 2007;5(2):164-169.

50. Graffam B. Metaphors we learn by [eletter]. http://www. annfammed.org/cgi/eletters/5/2/164\#5579, 4 April 2007.

51. Stange JP. Supportive communication: doctors are the band as well as improvisers [eletter]. http://www.annfammed.org/cgi/eletters/5/2/164\#5547, 1 April 2007.

52. Longenecker R. Improvisation and a seventh competency [eletter]. http://www.annfammed.org/cgi/eletters/5/2/164\#5529, 28 March 2007.

\section{Thank You, TRACK Participants}

The Annals of Family Medicine hosts an online discussion of articles called TRACK. Our sincere thanks to the following participants in this dialogue from April 2006 through March 2007. To read comments or add your voice to the discussion, visit http://www.annfammed.org.

Ann Fam Med 2007;5:272-273. DOI: 10.1370/afm.642.

Patricia Adam

Tim A. Ahles

Syed M. Ahmed

Virginia A. Aita

Mark J. Albanese

Stefano Alice

Richard E. Allen

A. A. W. Amarasinghe

Laura Anderko

Geoffrey A. Ankeney

Mary E. Arenberg

Rebecca A. Argenti

Macaran A. Baird

Nancy J. Baker

Shari L. Barkin

Eric B. Bass

David W. Bates

Nicholas J. Batley

Elizabeth A. Bayliss

John W. Beasley

Julie A. Becker

Ian M. Bennett

Robert E. Birth

Richard D. Blondell

Henry R. Bloom

Stephen P. Bogdewic

Hayden B. Bosworth

Howard Brody

Sharon B. Buchbinder
Heiner C. Bucher

David L. Buckeridge

Frederick M. Burkle, Jr

S. L. C.

A. John Campbell

Doug Campos-Outcalt

Mieke Cardol

Patricia A. Carney

Shannon Carson

James Alan Cave

Shelley Chang

William Cissell

Deborah J. Cohen

Maureen P. Corry

Barry V. Coutinho

Luke Cowie

Benjamin F. Crabtree

Frederic C. Craigie

Brian K. Crownover

Kathleen A. Culhane-Pera

Rada K. Dagher

Mary E. D'Alton

Terence M. Davidson

Francois S. de Brantes

Jan De Maeseneer

An De Sutter

William DeJong

D. Todd Detar

Neal Devitt
Jennifer E. DeVoe

Diana A. DiNatale

Frank J. Domino

Christopher F. Dowrick

David R. Duhon

Anne L. Dunlop

Carmel B. Dyer

A. E.

Mark Eisenberg

John Eng

Bery J. Engebretsen

Ronald M. Epstein

Mitchell Feldman

Berrylin J. Ferguson

Larry S. Fields

Kevin Fiscella

Martin Fortin

Peter Franks

John J. Frey, III

Avraham Friedman

Dominick L. Frosch

George E. Fryer

Gerald Gartlehner

Roy J. Gerard

Gilles Girard

Anthony N. Glaser

Park W. (Bill) Gloyd

Jerry Goddard

Henci L. Goer
Roberta E. Goldman

Isabelle Goupil-Sormany

Larry A. Green

Naomi D. Gregory

Martin C. Gulliford

Oye Gureje

David L. Hahn

Richard Hansen

Cynthia Haq

Diane Harper

John N. Harrison

Iona Heath

Margaret R. Helton

Larry Henderson

Cees M. P. M. Hertogh

John L. Hick

John Hickner

David U. Himmelstein

Joseph Hobbs

Sharona Hoffman

William E. Hogg

Oksana V. Hoile

Lisa D. Howley

Catherine Hudon

Susan Hughes

Russell D. Hull

Cynda A. Johnson

Neelima J. Kale

Gene A. Kallenberg 